\title{
Utility of 3D CT Rib Study in Presurgical Planning of Autologous Ear Graft for Pinna Reconstruction in Microtia
}

\author{
Sneha S. Zanje ${ }^{1} \quad$ Nikhil V. Kamat ${ }^{1}$ Kunal K. Taware ${ }^{1} \quad$ M L. Rokade ${ }^{1}$ Ashesh C. Bhumkar $^{2}$ \\ ${ }^{1}$ Department of Radiodiagnosis, Jupiter Scan Centre, Mumbai, \\ Address for correspondence Sneha Zanje, DNB, Department of \\ Maharashtra, India \\ 2 Bhumkar ENT Hospital, Mumbai, Maharashtra, India \\ Radiodiagnosis, Jupiter Scan Centre, Mumbai 400602, Maharashtra, \\ India , India (e-mail: snehaz0607@gmail.com).
}

Indian J Radiol Imaging 2021;31:586-595.

\begin{abstract}
Purpose The aim of this study was to demonstrate the utility of three-dimensional computed tomography (3D) CT rib study in presurgical planning to select the autologous rib cartilage graft for pinna reconstruction.

Materials and Methods Total of 35 patients of microtia for autologous rib graft from April 2017 to February 2020 were evaluated in this study. All patients had a plain lowdose multislice $\mathrm{CT}$ chest. The length of costal cartilages of sixth to ninth ribs bilaterally and width and height of sixth and seventh rib costal cartilage synchondrosis were measured in 3D reconstructed true size coronal images with best possible length displayed. All patients had high-resolution computed tomography (HRCT) temporal studies done to evaluate for associated anomalies in external canal, middle ear cavities, and inner ear structures. Eleven patients had simultaneous HRCT temporal bone done after plain $\mathrm{CT}$ chest and rest who had done recent prior study were reviewed without repetition of study.

Results There were 19 males and 16 females for 3D CT rib study. Average age of the participants was 16.5 years. The average width of synchondrosis of sixth and seventh rib was $15.4 \mathrm{~mm}$ on right side and $14.7 \mathrm{~mm}$ on left side, average height of synchondrosis was $28.5 \mathrm{~mm}$ on right side and $30.7 \mathrm{~mm}$ on left side. Average length of the eighth rib costal cartilage was $88.6 \mathrm{~mm}$ on the right side and $90.5 \mathrm{~mm}$ on the left side. Average

\section{Keywords}

- 3D CT VRT

- autologous rib graft

- microtia length of the ninth rib was $63.2 \mathrm{~mm}$ on the right side and $58.2 \mathrm{~mm}$ on the left side. Costal cartilage calcifications were present in 9 patients.

Conclusion Preoperative 3D CT rib study provides accurate measurements of rib stock for sculpting autologous ear graft.
\end{abstract}

\section{Key Messages}

Autologous rib costal cartilage graft is gold standard in microtia for pinna reconstruction because of acceptable aesthetic results, durability, and fewer complications. Pre- operative three-dimensional computed tomography (3D CT) rib study gives accurate measurements for harvesting adequate stock in sculpting autologous graft. Virtual surgery can be done as presurgical planning on true size 3D CT rib cage to determine the site and framework for graft that gives
DOI https://doi.org/ 10.1055/s-0041-1735866 ISSN 0971-3026.

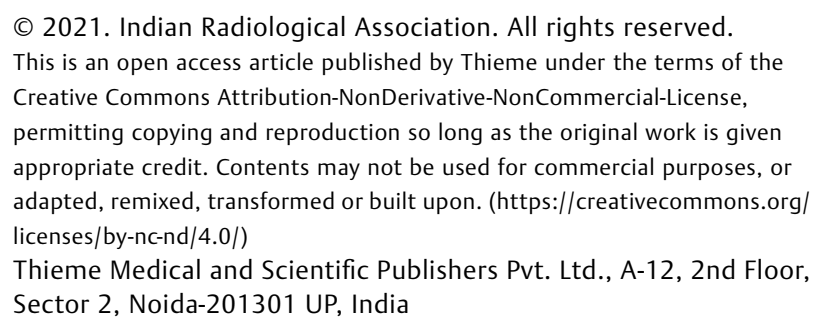


surgeons data to plan timing of surgery, consider modification in surgical technique, reduce surgical-time by anticipating on table surprises and possible complications with fair prediction for final outcome.

\section{Introduction}

Microtia is a congenital anomaly in which the external ear is either small or abnormal in shape. The estimated incidence of microtia is 1.8 per 10,000 live births, with a predilection for individuals of Hispanic, Asian, and Native American descent. ${ }^{1}$ Chest wall deformities have been reported to be higher in these individuals along with other associations like kidney anomalies. It can be associated with multiple syndromes; though unilateral occurrence in isolation is common. Incidence is higher in boys and on the right side. $^{2}$ In unilateral cases, contralateral ear appears normal in size, but detailed measurement studies have proven it to be smaller than the control groups. ${ }^{3}$ Microtia comprises variable severity of deformity involving pinna with variable anomalies of external acoustic canal (stenosis/atresia), middle ear structures, and occasionally inner ear. This leads to dual problems of cosmesis and hearing impairment of varying degrees.

Even though hearing correction is the most imperative factor among these patients, the aesthetic component of ear reconstruction cannot be undervalued due to associated psychosocial burden and stigma as children grow to school age where they face peer ridicule. ${ }^{4}$ Microtia surgery with auricular reconstruction is a unique blend to improve the cosmesis and functionality. Choice of optimal framework material to be used for graft and age of surgery are critical decisions for patients of microtia.

In our cohort of patients, a two-stage surgical approach using autologous rib cartilage was used for auricular reconstruction. A noncontrast CT of chest with 3D reconstruction of rib cage was done to evaluate costal cartilage growth, maturity, and calcification and associated anomalies. The CT scan for evaluation of rib cage in microtia patients has been underutilized. In this article, we highlight the evaluation of patients using 3D CT scan of rib cage to study various rib cartilage length and rib synchondrosis measurements. The status of costal cartilage calcification and associated anomalies of chest wall was evaluated. The purpose was to study to see the accuracy and assistance of this data in deciding surgical timing and selection of site of autologous rib cartilage graft for pinna reconstruction.

\section{Materials and Methods}

Between April 2017 AND February 2020, a total of 35 patients were done at Jupiter Imaging Centre with chest circumference measuring $60 \mathrm{cms}$ at xiphisternum. All these patients underwent low-dose plain multislice CT (MSCT) of chest with 3D reconstruction-MSCT rib study. The images were acquired using the GE bright speed 16 slice from the level of thyroid cartilage and covering all the ribs inferiorly. The imaging parameters are listed in -Table $\mathbf{1}$.
Table 1 Scanning parameters

\begin{tabular}{|l|l|}
\hline Scan type & Helical \\
\hline Detector configuration & $5 \mathrm{MM}, 16 \times 0.625$ \\
\hline FOV & $50 \mathrm{~cm}$ \\
\hline Pitch & $1.75: 1,1.375: 1$ \\
\hline Table speed & $17.5,13.75$ \\
\hline KVP & 100 \\
\hline mAs & Auto Mas \\
\hline Matrix & $512 \times 512$ \\
\hline
\end{tabular}

Abbreviation: FOV, field of view.

\section{Image Analysis}

Images were viewed using bone algorithm on GE workstation (AW volume share 4) with window width of 250 Hounsfield unit (HU) and window center $120 \mathrm{HU}$. The images were evaluated regarding length measurement of the sixth to ninth costal cartilages bilaterally and width and height of sixth and seventh costal cartilage synchondrosis bilaterally. The length of the costal cartilage was measured in 3D reconstructed coronal images with the best possible length displayed in the true image (-Figs. 1,2,3).

The medial landmark for length measurement in case of false eighth rib and ninth rib is the chondrosternal attachment for costal cartilage length. Note was made of costal cartilage calcification status and associated anomalies of chest wall like scoliosis, bifid ribs, supernumerary, or absent ribs and absent synchondrosis.

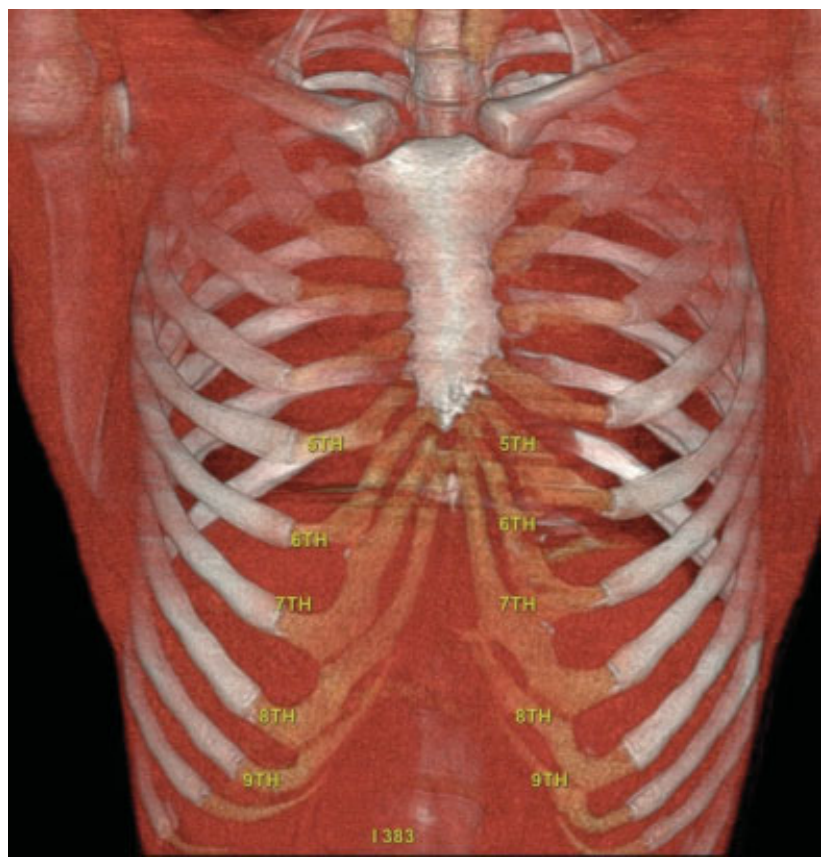

Fig. 1 A three-dimensional computed tomography volume-rendering technique TRUE size image displaying costal cartilage in its full length. This helps to evaluate shape, stock of cartilage, calcifications, and associated congenital anomalies to determine side for graft retrieval. 


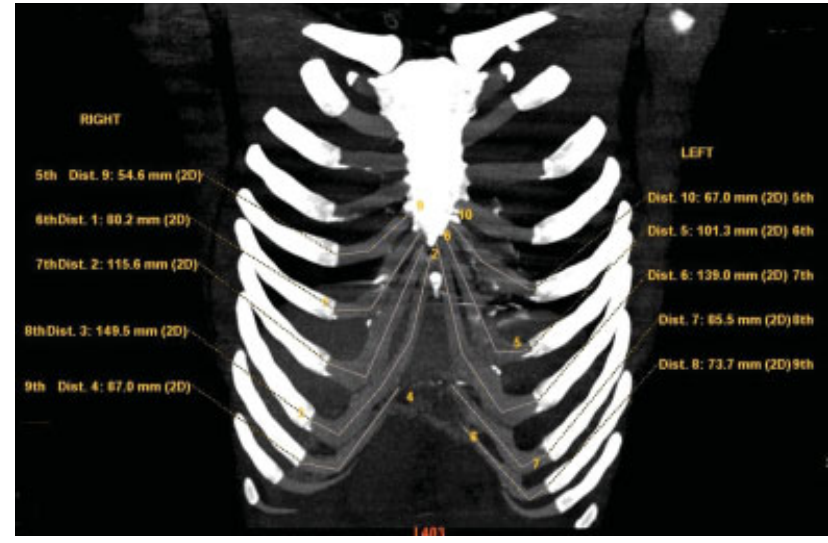

Fig. 2 Length of costal cartilage measured with best possible length displayed on three-dimensional coronal image. Landmark for length measurement was from costochondral junction laterally up to the medial end of the costal cartilage.

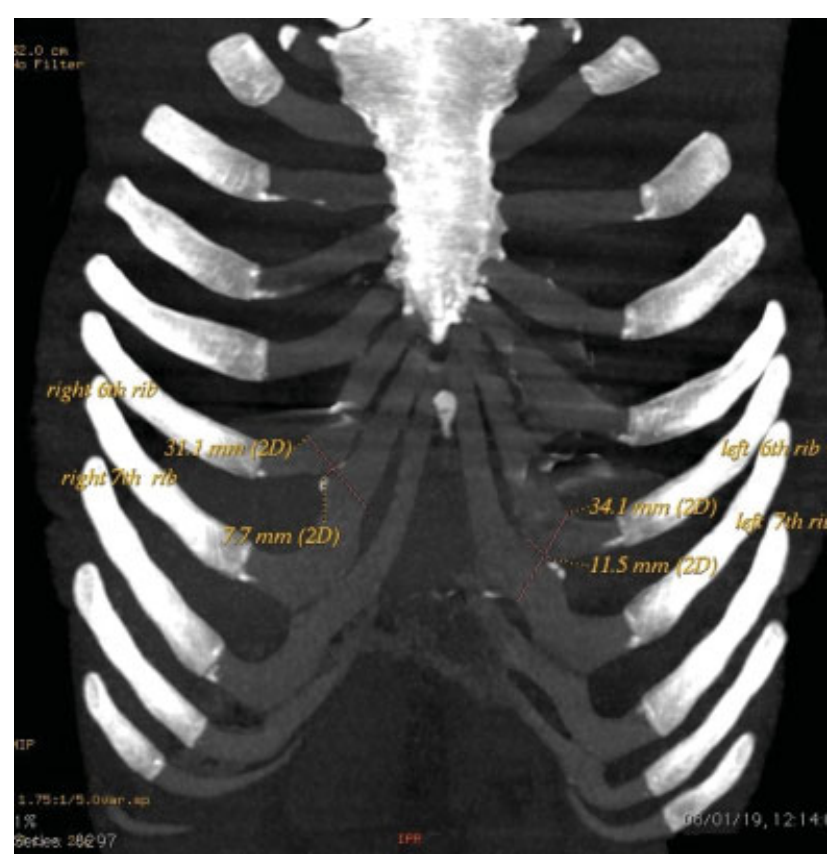

Fig. 3 Coronal three-dimensional SSD image displaying cartilage length is used for measuring height and width of 6th and 7th costal cartilage synchondrosis.

In our study, there were 19 male and 16 female patients. Average age of the patients was around 16.5 years. Calcifications were present in nine patients, and most commonly seen cartilage calcification pattern was marginal in five patients followed by central in two patients and granular in two patients.

The average width of synchondrosis of sixth and seventh costal cartilage was $15.4 \mathrm{~mm}$ on right side and $14.7 \mathrm{~mm}$ on left side ( - Graph 1 ), average height of synchondrosis on right side was $28.5 \mathrm{~mm}$ and on left side was $30.7 \mathrm{~mm}$ (-Graph 2). Average length of the eighth rib was $88.6 \mathrm{~mm}$ on the right side and $90.5 \mathrm{~mm}$ on the left side. Average length of the ninth rib was $63.2 \mathrm{~mm}$ on the right side and $58.2 \mathrm{~mm}$ on the left side ( - Tables $\mathbf{2}$ and $\mathbf{3}$ ).

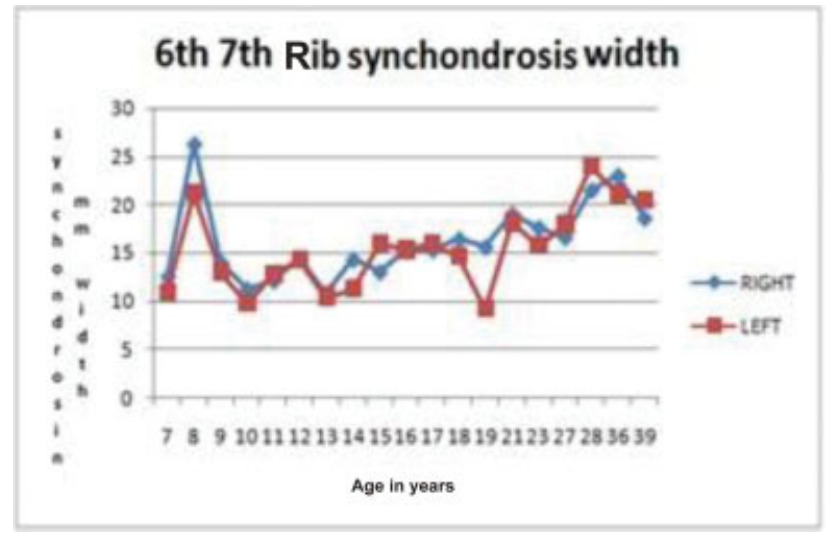

Graph 1 Growth curve of width of sixth and seventh rib synchondrosis in study subjects.

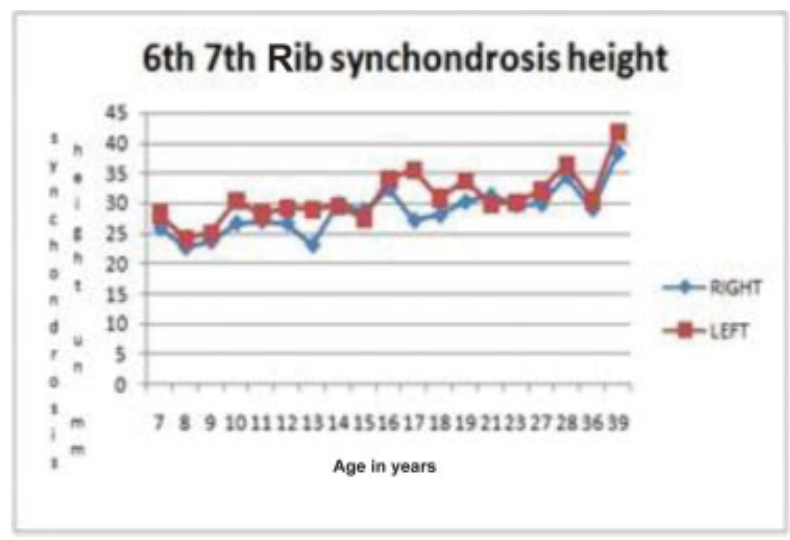

Graph 2 Growth curve of height of sixth and seventh rib synchondrosis in study subjects.

The growth of sixth and seventh ribs with costal cartilage was fairly linear up to 18 years, while the growth of eighth and ninth ribs is less consistent. Similarly, till 18 years and after 21 years, growth of sixth, seventh, eighth, and ninth ribs on right and left side was equal, whereas there was wide variation in the length between 18 and 21 years (-Graphs 3 and 4). The nonlinear growth of eighth and ninth ribs could be attributed to variation in detachment from sternum and transition into false rib. We have no justification for variation in length on left and right ribs between 18 and 21 years. With such small data, the finding may be fallacious and further larger study may throw light if the finding is true or false.

\section{Discussion}

Microtia is a congenital anomaly for the development of external ear; it can be a stand-alone anomaly or associated with multiple syndromes. ${ }^{2}$ Various methods have been used for ear reconstruction as described below. ${ }^{5}$

Artificial ear prosthesis with an osseointegrated titanium implant is an outpatient surgery with minimal morbidity and a preferred method in patients of trauma, cancer, and for salvage of autologous failures. $t$ has acceptable cosmetic 


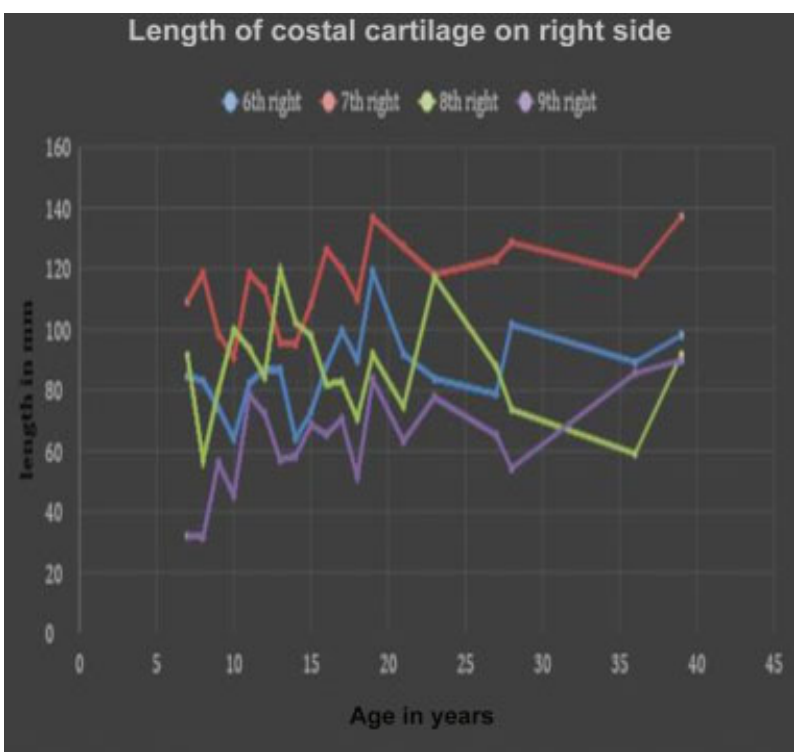

Graph 3 Growth based curves for the length of right sixth to ninth costal cartilage in study subjects.

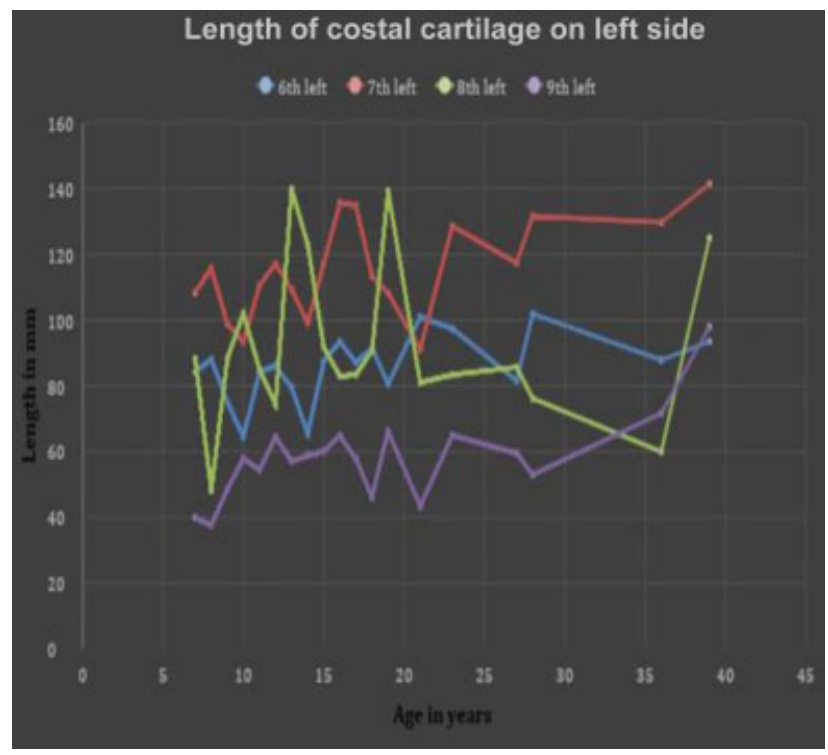

Graph 4 Growth based curve for the length of left sixth to ninth costal cartilage in study subjects.

result and surgery can be planned at any age. Selection of this method in children requires long-term commitment of both patient and prosthetic team. It requires ongoing expense of maintenance visits with future prosthesis changes every 2 to 5 years. In tropical countries like India, the sunlight and ultraviolet radiation can alter the color of prosthetic ears much earlier than in temperate countries warranting a much earlier prosthetic replacement. There is also a major challenge in maintaining hygiene and cleaning of the prosthesis and carries a risk of infection at the implantation site.

Medpore a synthetic biocompatible porous polyethylene implant pioneered by Reinisch has been used as an alternative to conventional autologous rib cartilage graft for ear reconstruction. ${ }^{5}$ This method uses a temporoparietal fascial flap to wrap the implant. A CT-angio can be performed preoperatively to see the vascularity of flap and to evaluate the caliber of superficial temporal artery and occipital artery of the flap area. Medpore has the ability to mimic the delicacy and projection of the normal opposite ear and is a one-stage outpatient procedure with a shorter learning curve with no need to wait for sufficient rib cartilage growth. Atresia repair in Medpore can be performed simultaneously with ear reconstruction or before the reconstruction. Medpore is an acellular foreign body with associated risk of implant extrusion, implant fracture, and soft tissue necrosis. The risk of breakdown of the covering skin can occur anytime in future thus having a potential risk of life-time complication. There is a probability of second surgery in cases of nonhealing wounds.

Autologous ear reconstruction is well-established and a popular method of surgery and remains mainstream of ear reconstruction surgery. It requires a great deal of surgical skill. Though a patient's own rib cartilage is utilized for graft, it is associated with relatively fewer complications and acceptable aesthetic results. These along with durability of the graft in long-term follow-up have contributed to its continuous success. Being a self-body structure, there are fewer chances of rejection than a foreign body. Also, autologous cartilage is a cellular structure and so is able to heal secondarily if exposed. This method has the advantage of ear reconstruction in anotia and secondary cases. The deterrent to this method includes possibility of chest deformities and multiple surgeries. Costal cartilage harvesting, subsequent thoracic wall scar, and deformity are necessary tradeoffs to obtain lifelong stability of reconstructed ears. Sculpting the cartilage framework for pinna is technically difficult. Yet due to several disadvantages of other methods using Medpore and osseointegrated prosthesis and many advantages of using self rib cartilage, the autologous rib cartilage for pinna reconstruction is considered gold standard in microtia ear repair surgery.

Significant disagreement exists regarding the timing of reconstruction surgery. Depending on the method used for fabrication of the auricular framework, the recommended age of reconstruction varies from 6 to 10 years. Tanzer ${ }^{6}$ introduced the autologous costal cartilage graft procedure. The Nagata ${ }^{7}$ technique for auricular reconstruction encompasses the two stages, the first is performed no earlier than 10 years of age and chest circumference at xiphoid level has grown to at least $60 \mathrm{~cm}$. Further modifications include Brent technique, Tunnel technique, and modified Nagata technique. ${ }^{8-14}$ Brent recommends surgery at 7 to 8 years of age before the children start to socialize and enter school to avoid peer ridicule and negative psychosocial impact. ${ }^{15}$ Chest wall deformity was seen in $8 \%$ of cases if costal cartilage was harvested between 6 and 12 years age and in 33\% if cases done between 2 and 3 years of age. ${ }^{16}$ In our part of the world due to lack of awareness of the corrective reconstruction surgeries and limited availability of reconstruction facilities, the average age of patients for reconstruction was 16.5 years that is higher than the recommended age in literature that is around 5 to 6 years for Medpore reconstruction and up to 10 years for autologous repair. 
The other factors impacting the timing of surgery are the psychosocial impact of microtia, impact of rib cartilage harvest on chest wall growth, and development and the further growth of the implanted graft.

Not many methods can be used to confirm the status of rib cartilage prior to surgery. Physical examination and chest Xray are done with limited results as these are unable to give information regarding the growth and maturity of cartilage and measure accurate length, thickness of cartilage along with the synchondrosis size.

A plain 3D CT chest provides highly accurate information about the costal cartilage. The use of rib cartilage measurements with reference to size of the healthy ear allows the initiation of ear reconstruction surgery as early as possible and helps in guiding the timing of auricular reconstruction to provide personalized treatment.

The growth of sixth and seventh rib cartilage shows a relatively linear pattern, whereas eighth rib cartilage shows variable pattern. ${ }^{17}$ This was also shown in our study. The cartilage state and stocks are important factors for harvesting. The formation of cartilage framework is a key element in the success of reconstruction surgery. Optimal reconstruction requires rib cartilage of sufficient size, length, and shape to sculpt a framework.

Attempts have been made by few study groups to utilize the $3 \mathrm{D}$ reconstructed images to compare rib cartilage preoperatively with intraoperative length, which proved its credibility. ${ }^{18-21}$ Meticulous measurements of the width and height of the synchondrosis of the sixth and seventh rib cartilages and the length of the eighth rib cartilage relative to the healthy ear provide guidance in planning the timing and process of surgical repair.

The size of synchondrosis is critical for the base of the framework. The base of the reconstructed ear framework was formed by splicing the synchondrosis of the sixth and seventh rib cartilage. The length of the eighth cartilage is one factor that determines the side used for the study. The eighth rib cartilage forms the helix in the reconstructed ear and determines the auricular outline. If the eighth rib cartilage is sufficiently long, the reconstructed helix can extend up to the lobule. If the eighth rib cartilage is shorter than the helix of the healthy ear, there will be a slender contour at the junction of the helix of the reconstructed ear to the lobule. The growth of the eighth rib cartilage is variable, so the length of the eighth rib cartilage is difficult to anticipate even with optimum chest diameter. The preoperative $\mathrm{CT}$ measurements of the eighth rib cartilage were shown to be well correlated with the actual length of the costal cartilage as measured intraoperatively.

The average length of eighth cartilage should be $7 \mathrm{~cm}$ to have good helix formation. If the length is insufficient, the cartilage from ninth to eleventh rib is utilized to fill the gap. However, this can sometimes give the reconstructed ear a fractured or notched appearance. This as a possible outcome of surgery can be explained preoperatively on the basis of CT images, so that patient has realistic expectations regarding the possible future appearance of the reconstructed ear.

Microtia reconstruction using autologous rib cartilage requires extensive presurgical planning. The presurgical challenges include the timing of the surgery, determining the side of graft (laterality), deciding technique, and predicting the possible complications and outcomes. A preoperative 3D CT rib study meets the challenges by giving accurate measurements that are required for harvesting adequate stock for sculpting of autologous graft, thereby reducing time of surgery.

Virtual surgery for customization and modification can be performed and planned preoperatively. This includes judging the adequacy of cartilage to give a good framework. The surgical expertise available for patients in our study has reduced the conventional four-stage surgery in two stages. In the first stage, auricular framework is harvested from the sixth to ninth cartilage, where the sixth and seventh synchondrosis is used to form the base of the framework with eighth and ninth cartilage being used to form helix and antihelix, respectively. Postoperative pressure bandage was given at rib harvest site to prevent hematoma. Antibiotics and keeping the wound site clean prevent infection. Pain management was done by catheter delivery of injection $0.5 \%$ bupivacaine and intravenous paracetamol. No special care is needed at auricular reconstruction site except for routine wound care to prevent infection. Second stage of surgery is usually done after 1 year and consists of elevation of the reconstructed auricle. In our setting, getting good lobule and maintaining symmetry to normal contra lateral ear was one of the major criteria for female patients prior to surgical reconstruction.

After a CT rib study is completed, an ear template ( - Fig. 4) of normal ear made on transparent plastic sheet or a preformed template for specific age group in case of bilateral involvement is placed on true size coronal film (-Fig. 5) as a guide for the amount of rib cartilage stock needed. Adequacy of length of cartilage is determined by length of cartilage displayed in the image.

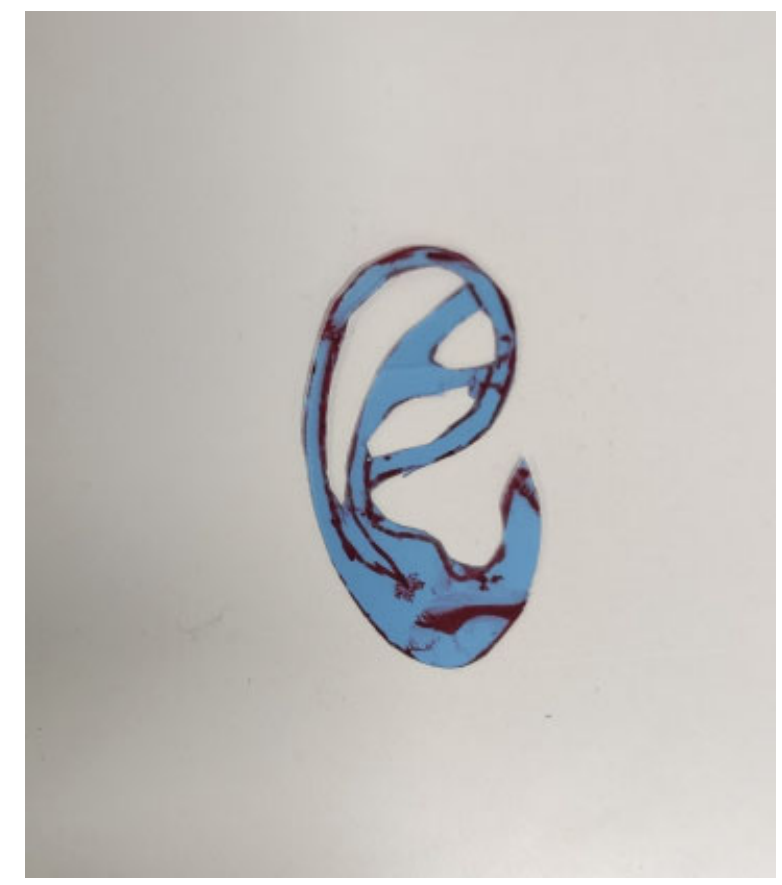

Fig. 4 Ear template constructed on transparent plastic sheet. 


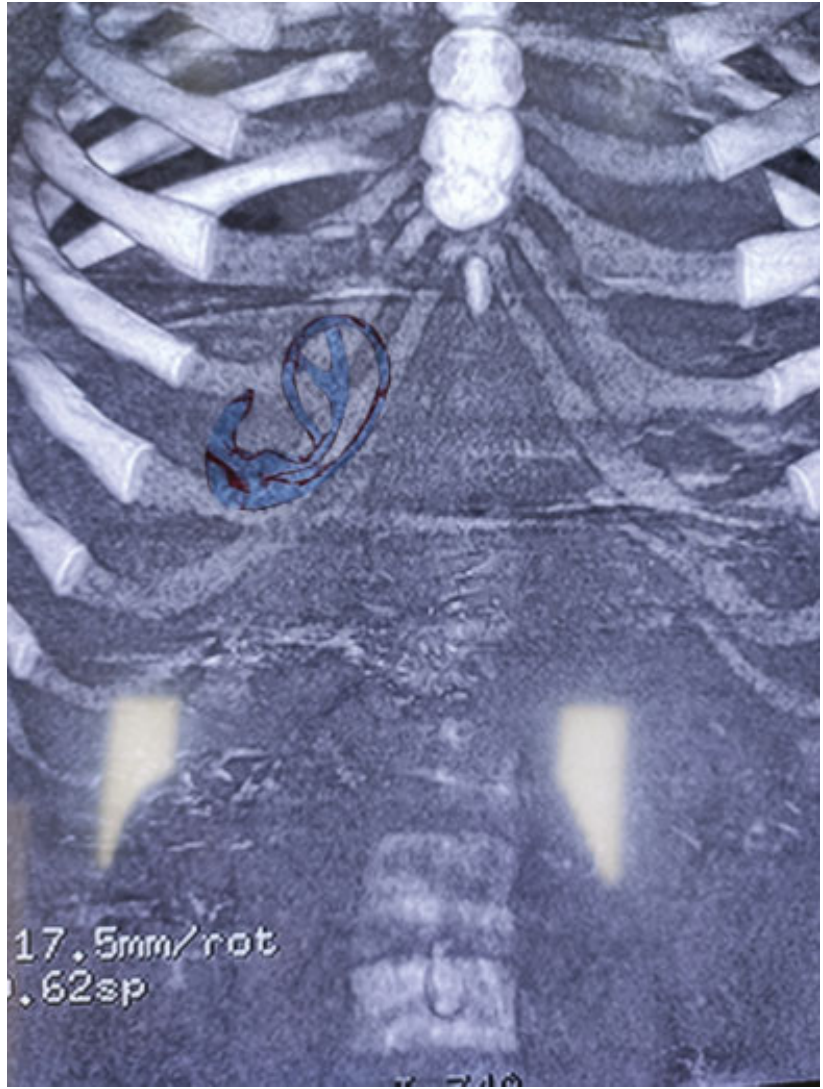

Fig. 5 Ear template kept on true size coronal image to evaluate cartilage stock for the base.

During first surgery after preplanning on virtual true sized coronal image, the ear template soaked in methylene blue dye is placed on the costal cartilage in vivo on thoracic cage (-Fig. 6) and then again on harvested sixth and seventh

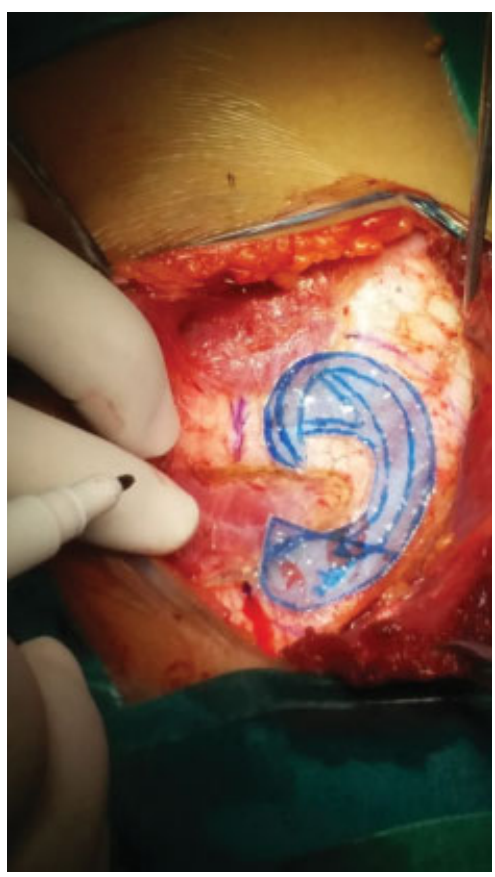

Fig. 6 Methylene blue-soaked template placed on rib cage at the site for harvesting.

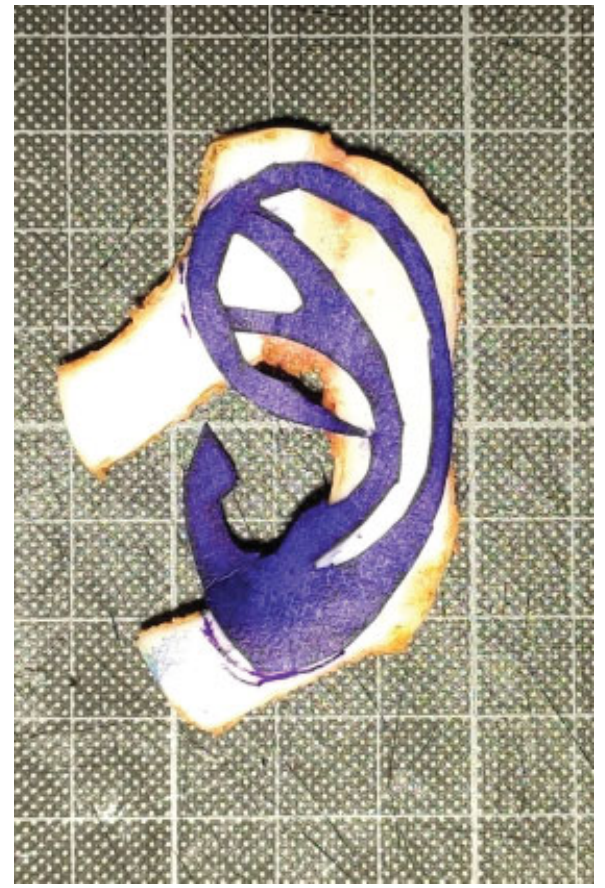

Fig. 7 Methylene blue-soaked template placed on harvested costal cartilage.

synchondrosis ( - Fig. 7). This base framework is attached and molded with eighth and eighth cartilage ( $\mathbf{- F i g s .} 8$ and $\mathbf{9}$ ) to form the antihelix and helix using steel wires and requires significant surgical skills and aesthetic refinement ( - Figs. 10 and 11).

One of the patients in our study had to undergo Medpore reconstruction due to inadequacy of cartilage stock in spite of fitting in criteria of more than $60 \mathrm{~cm}$ chest circumference at xiphoid level clinically. The true coronal 3D CT images revealed inadequate stock of the costal cartilage thus preventing an unnecessary surgical intervention (-Fig. 12A, 12B).

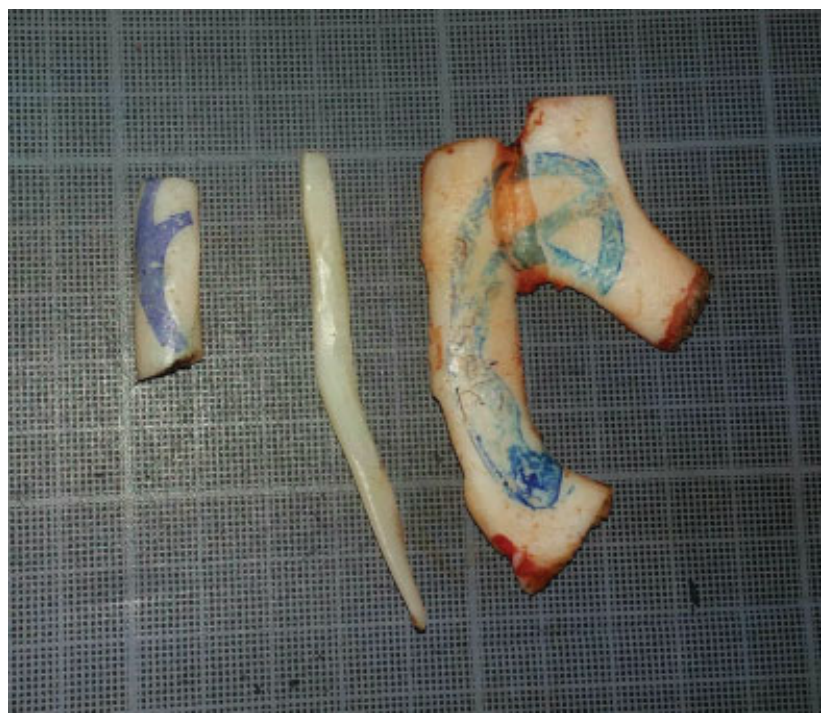

Fig. 8 Harvested framework formed by sixth to seventh synchondrosis with harvested eighth and ninth cartilage. 


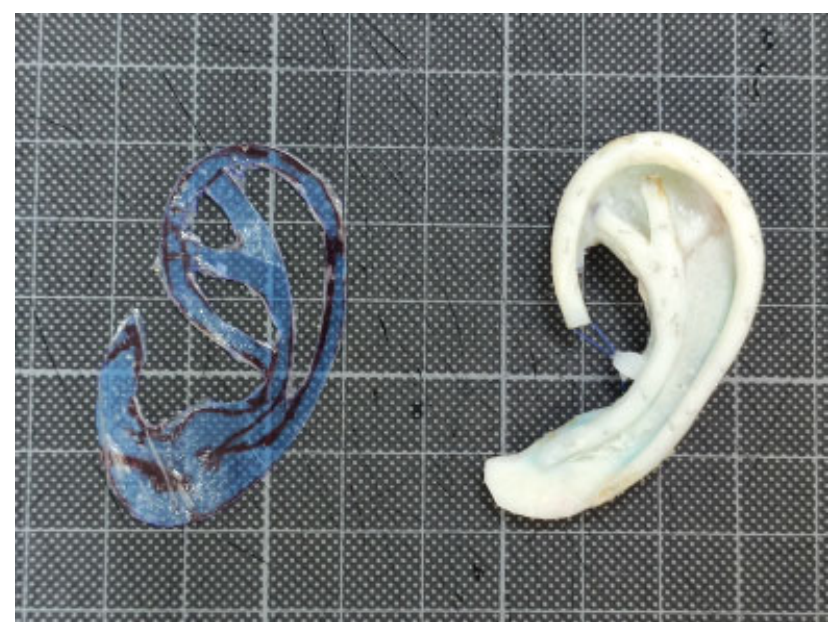

Fig. 9 Reconstructed ear template held in using steel wire.

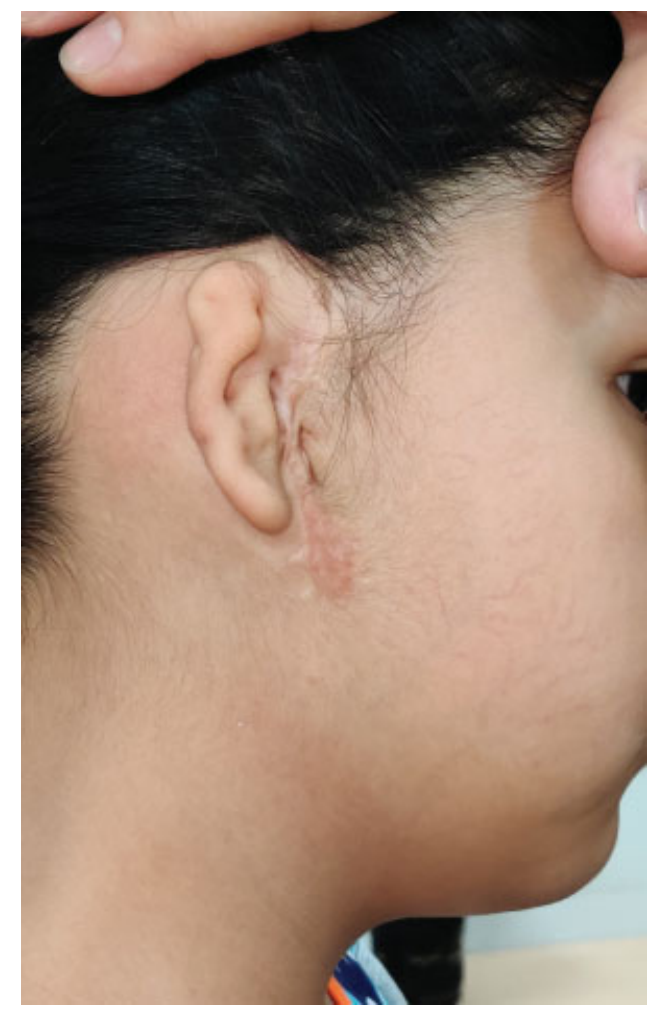

Fig. 10 Preoperative photograph of right ear showing severe microtia.

Presurgical imaging provides help in deciding laterality by comparing the symmetry and orientation of cartilage to the ear template. Location of calcification in images can decide laterality if they interfere at the site of the incision, as tissue engineering can become challenging in calcified tissue. Steel wires used for sculpting of the cartilage are difficult to manipulate in calcified cartilage. Calcified cartilage may be associated with slower healing and occasional complication of the resorption. A side with absent or lesser calcification is usually chosen. Costal cartilage calcification increases with age and is more common in female patients. 3D rib study has high sensitivity to detect the extent and pattern of calcification. Nine of our patients had patchy areas of calcification.

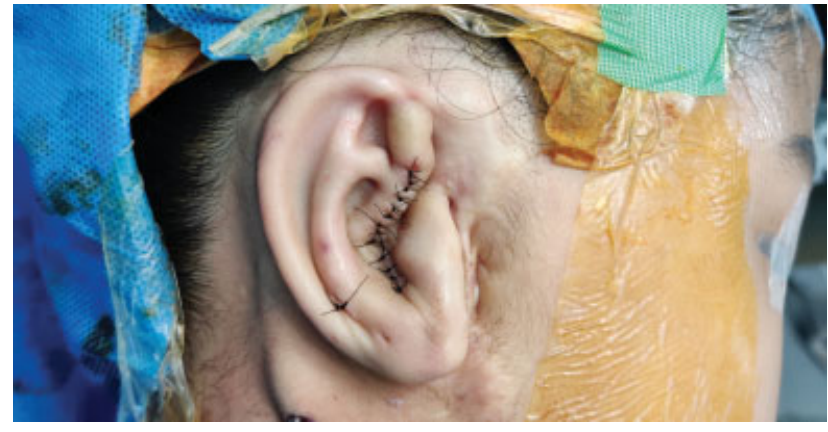

Fig. 11 Postoperative photograph of the same ear after placing autologous rib framework.
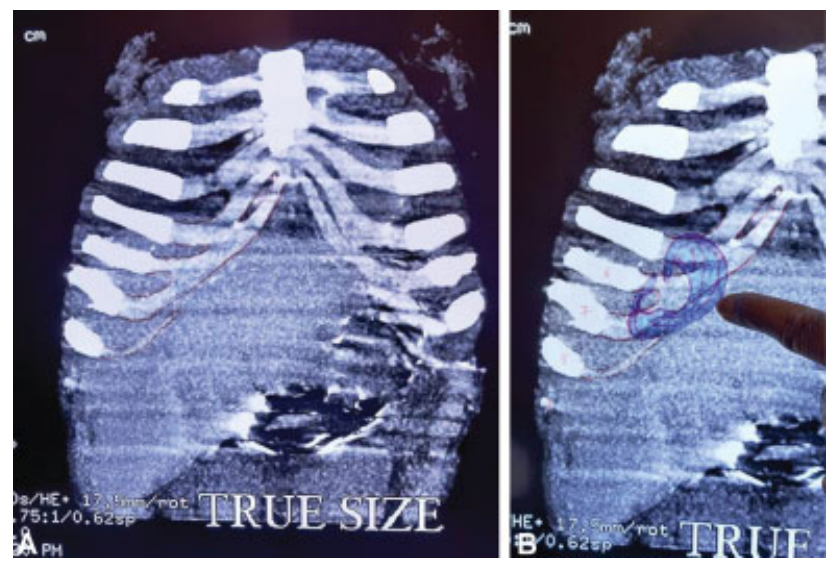

Fig. 12 (A) Presurgical computed tomography (CT) image revealing rib cartilage and its inadequacy in comparison with normal template. (B) Presurgical CT image revealing cartilage and its inadequacy in comparison with normal template.

Patients with heavily calcified bilateral cartilage have to undergo extensive splicing and sculpting of rib cartilage, leading to longer surgical time with slightly notched appearance of the graft. In such cases, the surgeon may favor a change in surgical technique like use of Medpore or prosthesis.

A 3D CT rib study can demonstrate associated congenital thoracic and rib deformities. Detection of anomalies like absent rib, bifid rib end, absent or unfused synchondrosis can help in selection of the most suited side of the rib cage. Only one of our patients had severe scoliosis of spine but no associated rib anomalies were seen. A thorough preoperative demonstration of rib cage can be done quickly, easily, efficiently, accurately on CT and knowledge of anomalies like pectus excavatum can prevent postsurgery chest wall complications and long-term deformities.

Magnetic resonance imaging (MRI) evaluation of costal cartilage protocols includes coronal short tau inversion recovery fast spin echo, coronal $\mathrm{T} 1$, and axial $\mathrm{T} 1$ images. T1-weighted images reveal normal anatomy and are useful for marrow evaluation in ossified regions. The coronal plane images tend to be the most effective in evaluation of costal cartilage pathologies and costal cartilage length in its entirety. The benefit of MRI is absence of radiation. However, it is protracted procedure that is marred by breathing and pulsation artifacts. Poor compliance is seen in pediatric age group 

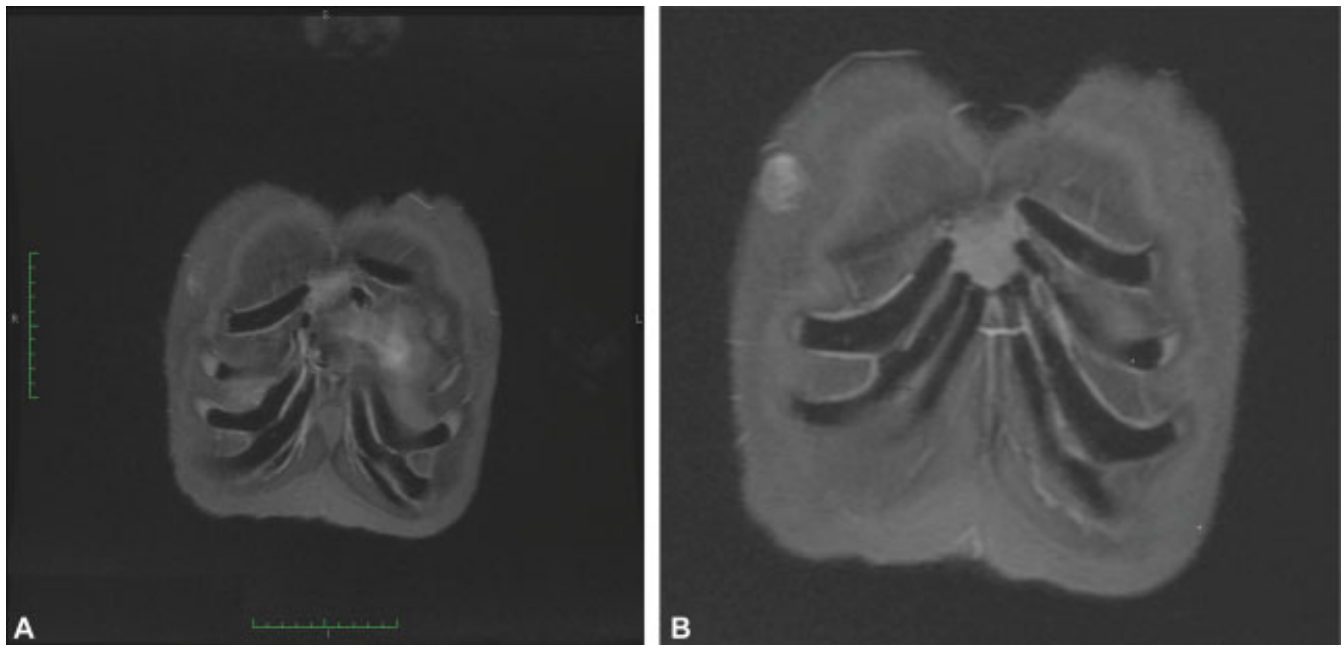

Fig. 13 (A) Magnetic resonance imaging coronal short tau inversion recovery (MRI COR STIR) displaying costal cartilage. (B) MRI COR STIR displaying costal cartilage.

Table 2 Length of costal cartilage as per the age of study subjects

\begin{tabular}{|l|l|l|l|l|l|l|l|l|}
\hline \multirow{2}{*}{ Age in years (no of patients) } & \multicolumn{2}{l|}{ 6th rib $(\mathbf{m m})$} & \multicolumn{2}{l|}{ 7th rib $(\mathbf{m m})$} & \multicolumn{2}{l|}{ 8th rib (mm) } & \multicolumn{2}{l|}{ 9th rib (mm) } \\
\cline { 2 - 9 } & Right & Left & Right & Left & Right & Left & Right & Left \\
\hline 7 & 84.5 & 84.2 & 109.3 & 108.4 & 91.3 & 88.2 & 32 & 39.9 \\
\hline 8 & 83.1 & 88.2 & 118.4 & 115.8 & 56.9 & 48.2 & 31.6 & 37.6 \\
\hline $9(3)$ & 74 & 75 & 98.4 & 98.9 & 80.1 & 88.3 & 56.2 & 48.6 \\
\hline $10(4)$ & 63.9 & 64.5 & 90.9 & 93.4 & 99.9 & 102 & 45.4 & 57.9 \\
\hline $11(3)$ & 82.3 & 84.3 & 118.3 & 110.6 & 93.7 & 85 & 78.3 & 54.5 \\
\hline $12(3)$ & 86.9 & 86.1 & 113 & 117.1 & 84.3 & 74.3 & 72.2 & 64.5 \\
\hline 13 & 86.7 & 79.5 & 95.8 & 109.4 & 119.6 & 139.9 & 57 & 57 \\
\hline $14(2)$ & 64 & 65.5 & 95.2 & 99.2 & 102.3 & 123 & 58.3 & 58.8 \\
\hline 15 & 72.8 & 87.4 & 108.3 & 117.3 & 97.8 & 91.8 & 68.6 & 60.1 \\
\hline $16(3)$ & 88 & 93.5 & 126.2 & 135.6 & 82 & 83 & 65.4 & 64.9 \\
\hline $17(2)$ & 99.6 & 87.1 & 119.9 & 134.9 & 82.7 & 83.4 & 70.5 & 57.9 \\
\hline 18 & 89.7 & 91.6 & 110 & 113.5 & 70.6 & 90.6 & 51.5 & 46.2 \\
\hline 19 & 119 & 80.6 & 136.6 & 108.2 & 91.8 & 139.3 & 83.5 & 66.2 \\
\hline 21 & 91.9 & 101 & 127.2 & 91.2 & 74.5 & 81 & 63.2 & 43.4 \\
\hline $23(2)$ & 83.8 & 97.4 & 118 & 128.6 & 117.3 & 83.4 & 77.6 & 65 \\
\hline $27(2)$ & 78.8 & 81.6 & 123 & 117.4 & 87.8 & 85.7 & 65.2 & 59.5 \\
\hline $28(2)$ & 101.5 & 101.9 & 128.6 & 131.6 & 73.6 & 76.2 & 54.3 & 53 \\
\hline 36 & 89.2 & 87.9 & 118.3 & 129.7 & 59.1 & 60 & 85.6 & 71.7 \\
\hline 39 & 98.1 & 93.5 & 137.1 & 141.5 & 91.7 & 125.0 & 89.9 & 98.0 \\
\hline
\end{tabular}

where the administration of anesthesia has its own disadvantage.

The length of costal cartilages from costochondral junction may not be precise on MRI. Calcification quantification is better appreciated on CT (-Fig. 13).

From surgeon's point of view single glance cartilage stock assessment is easier on a volume-rendering technique CT images or a multiplanar reformation reconstruction. Concomitant lung pathology can also be evaluated on CT. All these limitations along with logistic issues in doing MRI favored us to do CT chest that is faster and can be done alongside with high-resolution computed tomography temporal bone (-Fig. 13A and B).

\section{Conclusion}

Microtia surgery is technically challenging but optimum results can be extremely rewarding as it provides cosmesis 
Table 3 Height and width of 6th and 7th rib synchondrosis as per the age of study subjects

\begin{tabular}{|l|l|l|l|l|}
\hline \multirow{2}{*}{$\begin{array}{l}\text { Age in years } \\
\text { (no of patients in group) }\end{array}$} & \multicolumn{3}{|l|}{$\begin{array}{l}\text { 6th and 7th rib synchondrosis } \\
\text { width in mm }\end{array}$} & \multicolumn{2}{l|}{$\begin{array}{l}\text { 6th and 7th rib synchondrosis } \\
\text { height in mm }\end{array}$} \\
\cline { 2 - 5 } & Right & Left & Right & Left \\
\hline 7 & 12.5 & 10.8 & 26 & 28.2 \\
\hline 8 & 26.3 & 21.2 & 22.8 & 24.2 \\
\hline $9(3)$ & 14 & 13 & 23.8 & 25.1 \\
\hline $10(4)$ & 11.2 & 9.7 & 26.8 & 30.4 \\
\hline $11(3)$ & 12.1 & 12.7 & 27.1 & 28.2 \\
\hline $12(3)$ & 14.3 & 14.3 & 26.8 & 29.3 \\
\hline 13 & 10.8 & 10.4 & 23.2 & 29 \\
\hline $14(2)$ & 14.3 & 11.2 & 29.9 & 29.6 \\
\hline 15 & 13 & 15.9 & 28.9 & 27.4 \\
\hline $16(3)$ & 15.3 & 15.3 & 32.4 & 34.1 \\
\hline $17(2)$ & 15.3 & 16.0 & 27.25 & 35.5 \\
\hline 18 & 16.4 & 14.6 & 28.1 & 30.9 \\
\hline 19 & 15.6 & 9.2 & 30.3 & 33.7 \\
\hline 21 & 19 & 18.1 & 31.3 & 29.9 \\
\hline $23(2)$ & 17.6 & 15.7 & 29.7 & 30.2 \\
\hline $27(2)$ & 16.6 & 17.9 & 29.9 & 32.1 \\
\hline $28(2)$ & 21.5 & 24 & 34.4 & 36.5 \\
\hline 36 & 23 & 21.1 & 29.1 & 30.6 \\
\hline 39 & 18.6 & 20.5 & 38.3 & 41.8 \\
\hline
\end{tabular}

with hearing improvement. It eliminates the psychosocial stigma and reduces the hearing disability.

Autologous rib costal cartilage graft is considered gold standard in cases of microtia for pinna reconstruction due to its acceptable aesthetic results, durability, and fewer complications. A preoperative 3D CT rib study gives accurate measurements that are required for harvesting adequate stock for sculpting of autologous graft. Virtual surgery can be done as part of presurgical planning on a true size 3D CT rib cage to determine the site and framework for graft. It also gives surgeons the data to plan the timing of surgery, consider modification in surgical technique, reduce time for surgery by reducing on table surprises and hasten tissue engineering of autologous graft, anticipate possible complications, and predict the final outcome. ${ }^{22,23}$

\section{Source(s) of Support}

Nil.

\section{Presentation at a Meeting}

No.

\section{Financial Support and Sponsorship}

Nil.

\section{Conflicts of Interest}

There are no conflicts of interest.

\section{References}

1 Harris J, Källén B, Robert E. The epidemiology of anotia and microtia. J Med Genet 1996;33(10):809-813

2 Luquetti DV, Heike CL, Hing AV, Cunningham ML, Cox TC. Microtia: epidemiology and genetics. Am J Med Genet A 2012;158A (01):124-139

3 Matsuka K, Hata Y, Yano K, et al. Comparative study of auricular dimensions for the normal auricles of microtia patients, their parents, and normal individuals. Ann Plast Surg 1994;32(02):135-140

4 Steffen A, Wollenberg B, König IR, Frenzel H. A prospective evaluation of psychosocial outcomes following ear reconstruction with rib cartilage in microtia. J Plast Reconstr Aesthet Surg 2010; 63(09):1466-1473

5 Baluch N, Nagata S, Park C, et al. Auricular reconstruction for microtia: a review of available methods. Plast Surg (Oakv) 2014; 22(01):39-43

6 Tanzer RC. Total reconstruction of the external ear. Plast Reconstr Surg Transplant Bull 1959;23(01):1-15

7 Nagata S. A new method of total reconstruction of the auricle for microtia. Plast Reconstr Surg 1993;92(02):187-201

8 Brent B. Auricular repair with autogenous rib cartilage grafts: two decades of experience with 600 cases. Plast Reconstr Surg 1992; 90(03):355-374, discussion 375-376

9 Nagata S. Modification of the stages in total reconstruction of the auricle: Part I. Grafting the three-dimensional costal cartilage framework for lobule-type microtia. Plast Reconstr Surg 1994;93 (02):221-230, discussion 267-268

10 Nagata S. Modification of the stages in total reconstruction of the auricle: Part II. Grafting the three-dimensional costal cartilage framework for concha-type microtia. Plast Reconstr Surg 1994;93 (02):231-242, discussion 267-268 
11 Nagata S. Modification of the stages in total reconstruction of the auricle: Part III. Grafting the three-dimensional costal cartilage framework for small concha-type microtia. Plast Reconstr Surg 1994;93(02):243-253, discussion 267-268

12 Nagata S. Modification of the stages in total reconstruction of the auricle: Part IV. Ear elevation for the constructed auricle. Plast Reconstr Surg 1994;93(02):254-266, discussion 267-268

13 Zim SA. Microtia reconstruction: an update. Curr Opin Otolaryngol Head Neck Surg 2003;11(04):275-281

14 Ohara K, Nakamura K, Ohta E. Chest wall deformities and thoracic scoliosis after costal cartilage graft harvesting. Plast Reconstr Surg 1997;99(04):1030-1036

15 Brent B. Microtia repair with rib cartilage grafts: a review of personal experience with 1000 cases. Clin Plast Surg 2002;29(02):257-271, vii

16 Thomson HG, Kim TY, Ein SH. Residual problems in chest donor sites after microtia reconstruction: a long-term study. Plast Reconstr Surg 1995;95(06):961-968

17 Andreoli SM, Mills JC, Kilpatrick LA, White DR, Patel KG. CT measured normative cartilage growth in children requiring costochondral grafting. Otolaryngol Head Neck Surg 2013;149(06): 924-930
18 Moon IY, Oh KS, Lim SY, Pyon JK, Mun GH, Bang SI. Estimation of eighth costal cartilage in surgical timing of microtia reconstruction. J Craniofac Surg 2015;26(01):48-51

19 Kang SS, Guo Y, Zhang DY, Jiang DY. Rib cartilage assessment relative to the healthy ear in young children with microtia guiding operative timing. Chin Med J (Engl) 2015;128(16): 2208-2214

$20 \mathrm{Kim} \mathrm{H}$, Hwang JH, Lim SY, et al. Preoperative rib cartilage imaging in 3-dimensional chest computed tomography for auricular reconstruction for microtia. Ann Plast Surg 2014;72(04): 428-434

21 Wang Y, He L, Liu L, Chen W, Jiang H, Shang Q. [Evaluation of multi-slice spiral ct scan and image reconstruction technology in estimating costal cartilage volume]. Zhongguo Xiu Fu Chong Jian Wai Ke Za Zhi 2014;28(10):1266-1269

22 Costal Cartilage Injuries [Internet]. Radsource. 2020 [cited 2020 Nov 07]. Accessed August 18, 2021 from: https://radsource.us/costal-cartilage-injuries

23 Subhas N, Kline MJ, Moskal MJ, White LM, Recht MP. MRI evaluation of costal cartilage injuries. AJR Am J Roentgenol 2008;191 (01):129-132 